\title{
Using the EAGLE simulations to elucidate the origin of disc surface brightness profile breaks as a function of mass and environment
}

\author{
Joel L. Pfeffer, ${ }^{1 \star}$ Kenji Bekki, ${ }^{1}$ Duncan A. Forbes, ${ }^{2}$ Warrick J. Couch, ${ }^{2}$ Bärbel S. Koribalski ${ }^{3,4}$ \\ ${ }^{1}$ International Centre for Radio Astronomy Research (ICRAR), M468, University of Western Australia, 35 Stirling Hwy, Crawley, WA 6009, Australia \\ ${ }^{2}$ Centre for Astrophysics \& Supercomputing, Swinburne University of Technology, Hawthorn VIC 3122, Australia \\ ${ }^{3}$ Australia Telescope National Facility, CSIRO Astronomy and Space Science, P.O. Box 76, Epping, NSW 1710, Australia \\ ${ }^{4}$ School of Science, Western Sydney University, Locked Bag 1797, Penrith, NSW 2751, Australia
}

Accepted XXX. Received YYY; in original form ZZZ

\begin{abstract}
We analyse the surface brightness profiles of disc-type galaxies in the EAGLE simulations in order to investigate the effects of galaxy mass and environment on galaxy profile types. Following observational works, we classify the simulated galaxies by their disc surface brightness profiles into single exponential (Type I), truncated (Type II) and anti-truncated (Type III) profiles. In agreement with previous observation and theoretical work, we find that Type II discs result from truncated star-forming discs that drive radial gradients in the stellar populations. In contrast, Type III profiles result from galaxy mergers, extended star-forming discs or the late formation of a steeper, inner disc. We find that the EAGLE simulations qualitatively reproduce the observed trends found between profile type frequency and galaxy mass, morphology and environment, such as the fraction of Type III galaxies increasing with galaxy mass, and the the fraction of Type II galaxies increasing with Hubble type. We investigate the lower incidence of Type II galaxies in galaxy clusters, finding, in a striking similarity to observed galaxies, that almost no S0-like galaxies in clusters have Type II profiles. Similarly, the fraction of Type II profiles for disc-dominated galaxies in clusters is significantly decreased relative to field galaxies. This difference between field and cluster galaxies is driven by star formation quenching. Following the cessation of star formation upon entering a galaxy cluster, the young stellar populations of Type II galaxies simply fade, leaving behind Type I galaxies.
\end{abstract}

Key words: galaxies: structure - galaxies: disc - galaxies: formation - galaxies: evolution - methods: numerical

\section{INTRODUCTION}

The structural properties of galaxies are intimately linked to their formation and assembly. Originally thought to have exponential profiles (Patterson 1940; de Vaucouleurs 1959), the stellar surface brightness profiles of disc galaxies have now been shown to occur in three main types: single exponential (Type I), truncated (down-bending, Type II) and anti-truncated (up-bending, Type III) profiles (Freeman 1970; van der Kruit 1979; Erwin et al. 2005; Pohlen \& Trujillo 2006).

Though the exact fractions of disc types differ between studies (possibly due to differing galaxy samples or methodology, c.f. Méndez-Abreu et al. 2017), some general trends with galaxy mass and morphology are clear. With increasing galaxy mass, the fraction of Type I profiles decreases and the fraction of Type III profiles increases (Laine et al. 2016; Tang et al. 2020). The frequency of Type II galaxies increases with Hubble type (i.e. are more frequent in disc-dominated galaxies), while Type III profiles are more common in early-type galaxies (Pohlen \& Trujillo 2006; Gutiérrez et al. 2011; Laine et al. 2016; Méndez-Abreu et al. 2017; Tang et al. 2020). Approximately 8 per cent of galaxies also have composite Type II+III profiles (Gutiérrez et al. 2011).

In Type II (truncated) galaxies, the break in the exponential light

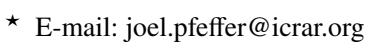

profile has been observed to coincide with a minimum in the colour, age and mass-to-light ratio profiles (Azzollini et al. 2008a; Bakos et al. 2008; Zheng et al. 2015; Ruiz-Lara et al. 2016). The breaks are largely absent in the mass profiles of Type II galaxies (Bakos et al. 2008; Tang et al. 2020) and are thus related to radial gradients in the stellar populations (similarly found in the simulations of SánchezBlázquez et al. 2009). The disc breaks may result from star formation thresholds in the outer parts of galaxies (e.g. Kennicutt 1989; Schaye 2004), and simulations of disc galaxies have found that the break may be a result of a star formation cutoff associated with a drop in cold gas density (Roškar et al. 2008; Martínez-Serrano et al. 2009; Sánchez-Blázquez et al. 2009). This picture is consistent with the down-bending star-formation rate (SFR) profiles of Type II galaxies (Tang et al. 2020). The radial position of the break has also been found to increase with cosmic time (Trujillo \& Pohlen 2005; Azzollini et al. 2008b) and galaxy mass (Pohlen \& Trujillo 2006; Muñoz-Mateos et al. 2013), consistent with inside-out growth of disc galaxies.

A number of origins have been suggested for Type III (antitruncated) galaxies. They may be the result of galaxy mergers (e.g. Bekki 1998; Bournaud et al. 2005), other tidal disturbances or galaxy harassment (Watkins et al. 2019), star formation from accreted gas (Roediger et al. 2012; Wang et al. 2018), stellar migration induced by a long-lived bar (Herpich et al. 2017) or the combination of a pseudo-bulge and an outer Type I disc (Sil'chenko et al. 2018). This 
diversity of origins may be reflected in the structure of the galaxies, where some anti-truncations may be the result of a disc embedded within an outer spheroid, while others are a continuation of the disc (Erwin et al. 2005). Type III galaxies have been found to have upbending SFR profiles, though without strong radial gradients in the stellar populations (i.e. the surface brightness and mass profiles are closely related in Type III galaxies, Tang et al. 2020). Borlaff et al. (2018) found Type III break radius scaling relations are similar from $z=0.6$ to $z=0$, indicating a 'stable' formation process.

A curious and so far, unexplained, observation is that, despite being common for field $\mathrm{S} 0$ galaxies, Type II profiles are completely absent for S0 galaxies in the Virgo cluster (Erwin et al. 2012). Roediger et al. (2012) and Raj et al. (2019) similarly found that the fraction of Type II profiles in Virgo and Fornax cluster disc galaxies are suppressed relative to field galaxies (which decrease from 50-60 per cent in field galaxies to 34 per cent in the Virgo cluster and 38 per cent in the Fornax cluster, respectively). Lee et al. (2018) also found a similar environmental dependence for Type II profiles of dwarf galaxies in the Virgo cluster and NGC 2784 group. The origin of this difference is not yet understood, but might plausibly be driven by different formation or evolutionary processes in field and cluster environments.

In this work, we analyse the mass and surface brightness profiles of disc-type galaxies in the EAGLE simulations (Schaye et al. 2015; Crain et al. 2015) in order to investigate the effect of environment on galaxy profile types, with the aim of understanding the suppressed fraction of Type II galaxies in galaxy clusters. In Section 2 we describe the EAGLE simulations and analysis of the simulations. In Section 3 we first discuss the origin of Type II and III profiles in EAGLE galaxies, before exploring the the effect of environment on disc type frequencies in Section 4. Finally, we summarise the results in Section 5.

\section{METHODS}

\subsection{EAGLE simulations}

The Evolution and Assembly of GaLaxies and their Environments (EAGLE) simulations are a suite of cosmological, hydrodynamical simulations of galaxy formation in the $\Lambda$ cold dark matter $(\Lambda \mathrm{CDM})$ cosmogony (Schaye et al. 2015; Crain et al. 2015). The simulations were run with a highly modified version of the $N$-body TreePM smooth particle hydrodynamics (SPH) code GADGET3 (last described by Springel 2005), with updates to the SPH formulation, time-stepping and subgrid physics (see appendix A of Schaye et al. 2015). EAGLE includes subgrid routines describing radiative cooling (Wiersma et al. 2009a), star formation (Schaye \& Dalla Vecchia 2008), stellar evolution and mass loss (Wiersma et al. 2009b), the seeding and growth of black holes (BHs) via gas accretion and $\mathrm{BH}-$ BH mergers (Rosas-Guevara et al. 2015), and feedback associated with star formation (Dalla Vecchia \& Schaye 2012) and BH growth (Booth \& Schaye 2009). The simulations adopt cosmological parameters consistent with a Planck Collaboration (2014) cosmology, namely $\Omega_{\mathrm{m}}=0.307, \Omega_{\Lambda}=0.693, \Omega_{\mathrm{b}}=0.04825, h=0.6777$ and $\sigma_{8}=0.8288$. Dark matter structures are first identified by running the friends-of-friends (FOF) algorithm (Davis et al. 1985) with a linking length 0.2 times the mean interparticle separation. Bound galaxies (subhaloes) were identified within the FOF groups using the SUBFIND algorithm (Springel et al. 2001; Dolag et al. 2009).

The stellar and $\mathrm{BH}$ feedback parameters are calibrated such that the simulations of cosmologically representative volumes reproduce the galaxy stellar mass function, galaxy sizes and $\mathrm{BH}$ masses at $z \approx 0$ (Crain et al. 2015). Following this the EAGLE simulations reproduce a large range of observed galaxy population properties, making them ideal for comparisons with observed galaxy populations, including: the evolution of the galaxy stellar mass function (Furlong et al. 2015) and sizes (Furlong et al. 2017), galaxy luminosities and colours (Trayford et al. 2015), galaxy morphologies (Bignone et al. 2020), cold gas properties (Lagos et al. 2015, 2016; Bahé et al. 2016; Marasco et al. 2016; Crain et al. 2017), and the properties of circumgalactic and intergalactic absorption systems (Rahmati et al. 2015, 2016; Oppenheimer et al. 2016; Turner et al. 2016, 2017), while broadly reproducing the cosmic star formation rate density and relation between specific star formation rate and galaxy mass (Furlong et al. 2015).

This work makes use of both the EAGLE reference simulation (Ref-L100N1504) and the higher resolution 'recalibrated' simulation (Recal-L025N0752; see Schaye et al. 2015 for further details of the simulations). EAGLE Ref-L100N1504 simulates a periodic volume with side length of 100 comoving Mpc using $1504^{3}$ gas and dark matter particles with initial baryonic masses $1.81 \times 10^{6} \mathrm{M}_{\odot}$ and maximum gravitational softening length $0.7 \mathrm{kpc}$. EAGLE RecalL025N0752 simulates a periodic volume with a side length of 25 comoving Mpc using $752^{3}$ gas and dark matter particles with initial baryonic masses $2.26 \times 10^{5} \mathrm{M}_{\odot}$ and maximum softening length $0.35 \mathrm{kpc}$.

\subsection{Sample selection and analysis}

We selected galaxies to have disc-type morphologies using the parameter $\kappa_{\mathrm{co}}$, the fraction of stellar kinetic energy invested in ordered co-rotation (Correa et al. 2017; Thob et al. 2019). Correa et al. (2017) found that low $\kappa_{\mathrm{co}}$ galaxies $\left(\kappa_{\mathrm{co}} \sim 0.2\right)$ tend to be spheroidal-shaped galaxies, while high $\kappa_{\mathrm{co}}$ galaxies $\left(\kappa_{\mathrm{co}} \sim 0.7\right)$ tend to be disc-shaped galaxies. Following Correa \& Schaye (2020), we select disc-type galaxies to have $\kappa_{\mathrm{co}}>0.35$. With this selection, our sample ranges from S0s $\left(\kappa_{\mathrm{co}} \sim 0.4\right)$ to late-type disc $\left(\kappa_{\mathrm{co}} \gtrsim 0.6\right)$ galaxies (Correa et al. 2019).

In order to sample the radial profiles of the galaxies, we only consider well resolved galaxies with stellar masses $M_{*}>10^{10} \mathrm{M}_{\odot}$, corresponding to galaxies with $>5 \times 10^{3}$ stellar particles in the Ref-L100N1504 simulation and $>4 \times 10^{4}$ particles in the RecalL025N0752 simulation. We exclude galaxies in close proximity to significant neighbouring galaxies which result in major disturbances in their surface brightness profiles at the position of the neighbour galaxy, or obvious truncations resulting from the SUBFIND algorithm and the lack of density contrast between the substructures. This selection gives us a sample of 2290 galaxies for the reference simulation and 50 galaxies for the high resolution simulation.

For comparison with observed galaxy luminosity profiles (i.e. Erwin et al. 2012), we convert stellar mass profiles of the simulated galaxies to SDSS $r$-band profiles using the FSPS stellar population model (Conroy et al. 2009; Conroy \& Gunn 2010) assuming a Chabrier (2003) initial stellar mass function and using the Miles spectral library (Sánchez-Blázquez et al. 2006) and Padova isochrones (Girardi et al. 2000; Marigo \& Girardi 2007; Marigo et al. 2008). We assume simple stellar populations for each stellar particle, and determine mass-to-light ratios for each particle by linearly interpolating from the grid in ages and total metallicities. As a simple model for dust absorption, we assume stars with ages $<10 \mathrm{Myr}$ are fully embedded within an optically thick cloud (e.g. Charlot \& Fall 2000).

Before classifying the $r$-band profiles we rotated all galaxies into face-on projections, such that they are comparable with face-on to 
moderately inclined samples of observed galaxies (e.g. Pohlen \& Trujillo 2006; Erwin et al. 2008; Gutiérrez et al. 2011; MuñozMateos et al. 2013; Laine et al. 2014; Tang et al. 2020). To rotate the galaxies we calculate the spin vector for all stars between 2.5 and $30 \mathrm{kpc}$ from the centre of potential each the galaxy. The radius limits were chosen following Trayford et al. (2017), such that the spin is dominated by the rotating disc component of the galaxy, if present, rather than bulge regions.

Radial surface brightness profiles for each galaxy were then created by using circular annuli about the centre of potential of the galaxy. We use linearly increasing annuli, where the distance between inner and outer radii increases linearly with each annulus, beginning at $0.1 \mathrm{kpc}$ and increasing in steps by $0.02 \mathrm{kpc}$, reaching a width of $1 \mathrm{kpc}$ at a radius of $\approx 25 \mathrm{kpc}$. In this way the the densest, inner regions of each galaxy can be reasonably resolved, while retaining annuli in the outskirts of galaxies with areas large enough to avoid stochasticity due to particle resolution.

We classified the $r$-band surface brightness profile of each galaxy visually into exponential (Type I), down- (Type II) and up-breaking (Type III), and composite profiles (e.g. Type II break followed by a Type III break, or the inverse), similar to the manor applied in observational works (e.g. Erwin et al. 2005; Pohlen \& Trujillo 2006; Erwin et al. 2008). We adopt an $r$-band surface brightness limit of $27 \mathrm{mag} \mathrm{arcsec}^{-2}$ for classifying the profiles, similar to observations in Erwin et al. (2012). In cases where multiple profile breaks are found (composite profiles) we adopt the break type at the smallest galactocentric radius, as an outer break might often fall below the surface brightness limit if a brighter limit was adopted (i.e. $<27 \mathrm{mag} \mathrm{arcsec}^{-2}$ ).

\section{ORIGIN OF DISC PROFILE BREAKS IN EAGLE GALAXIES}

In this section, we first investigate the origin of disc profile breaks with the Recal-L025N0752 simulation, which better samples the radial profiles due to its higher resolution.

\subsection{Type I: exponential disc}

As a reference to contrast with the truncated (Section 3.2) and antitruncated (Section 3.3) galaxy profiles, we first show an example of a Type I galaxy from the EAGLE Recal-L025N0752 simulation in Fig. 1. The surface brightness profile shows a clear inner bulge (well fit by a Sérsic 1963 profile), as well as an outer exponential disc. The galaxy shows very similar light and mass profiles (upper panel), due to its very flat mass-to-light ratio profile (second panel).

The evolution of the density profile for the galaxy in is shown in the bottom panel of Fig. 1. The bulge of the galaxy forms by $z \sim 1.5$, while the disc continues to form to $z \approx 0.2$. The evolution of the density profile for this galaxy is relatively smooth due to the absence of any significant galaxy accretion events. The truncated profile at $z=0.1$ occurs where the subFIND algorithm assigns the outer particles of this galaxy to a more massive galaxy during a close passage in a galaxy group (see Knebe et al. 2011 and Muldrew et al. 2011 for further discussion on the difficulties of subhalo detection in a high background density). These outer particles are reassigned to the galaxy in the $z=0$ snapshot. As a result of the interaction, the galaxy has been stripped of most of its gas beyond $5 \mathrm{kpc}$ at $z=0$ (third panel).

We note that not all Type I galaxies follow identical formation histories. We find examples of Type I galaxies that form outside-in,
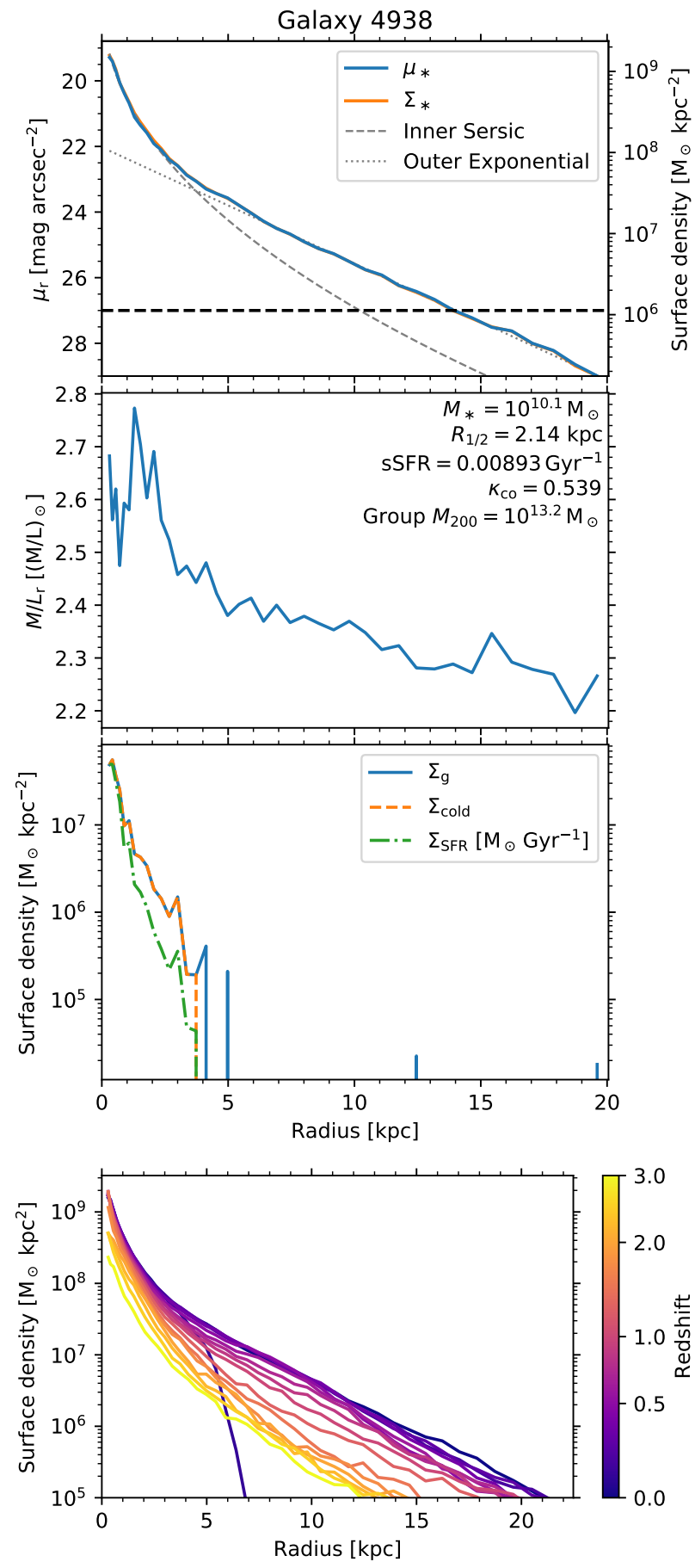

Figure 1. Example of a Type I galaxy from the EAGLE Recal-L025N0752 simulation (GalaxyID=4938). The top panel shows the projected $r$-band surface brightness (solid blue line, left axis) and mass density profiles (solid orange line, right axis). The horizontal dashed line shows the adopted surface brightness limit of $27 \mathrm{mag} \mathrm{arcsec}{ }^{-2}$. The grey dashed line shows a Sérsic (1963) profile fit to the inner $2.5 \mathrm{kpc}$ of the surface brightness profile, while the grey dotted line shows an exponential profile fit to the outer quarter of profile (up to $27 \mathrm{mag} \operatorname{arcsec}^{-2}$ ). The second panel shows the radial profile of the $r$-band mass-to-light ratio. Text in the second panel displays information about the galaxy (i.e. stellar mass, half-mass radius, specific star formation rate, kinematic morphology $\left(\kappa_{\mathrm{co}}\right)$ and group $\left.M_{200}\right)$. The third panel shows the surface density profiles for the total gas mass $\left(\Sigma_{\mathrm{g}}\right)$, star-forming (cold) gas mass $\left(\Sigma_{\text {cold }}\right)$ and star formation rate $\left(\Sigma_{\mathrm{SFR}}\right.$, in $\left.\mathrm{M}_{\odot} \mathrm{Gyr}^{-1} \mathrm{kpc}^{-2}\right)$. The bottom panel shows the evolution of the mass surface density profile for the galaxy from $z=3$ to $z=0$. 
as well as those that retain a Type I profile despite significant late galaxy mergers.

\subsection{Type II: truncated disc}

We show two examples of EAGLE galaxies with truncated disc profiles in Figures 2 (though the results apply more generally to all Type II galaxies found in the simulation). Based on their kinematic morphology $\left(\kappa_{\mathrm{co}}\right)$, both examples would be considered spiral galaxies. For both galaxies, the Type II break in the disc profiles $\left(R_{\text {break }} \approx 14 \mathrm{kpc}\right.$ for Galaxy $697075, R_{\text {break }} \approx 12 \mathrm{kpc}$ for Galaxy 971267) occurs near the minimum in the mass-to-light ratio profiles (both galaxies show the characteristic ' $\mathrm{U}$ ' shaped $M / L$ profiles). In both cases, the low mass-to-light ratios are due to the younger stellar populations near the disc break (median stellar particle ages $\sim 2$ Gyr younger than the galaxy as a whole). Beyond the break radii, the mass-to-light ratios increase due to the transition to older, more metal-poor stellar populations (i.e. the stellar halo).

In both galaxies, the minimum in the $M / L$ ratio profile is driven by a rapid drop-off in the cold gas mass and SFR beyond the disc break radius (third panels from the top). The cold gas mass and SFR density show a drop-off once the total gas surface density falls below $\sim 10^{6.5} \mathrm{M}_{\odot} \mathrm{kpc}^{-2}$, which result from the density threshold for star formation used in the EAGLE star formation model (Schaye 2004; Schaye \& Dalla Vecchia 2008). Beyond the drop-off in the cold gas density/SFR, the mass-to-light ratio of the stellar population rises, thus creating the appearance of a 'break' in the disc profile.

In the case of Galaxy 697075 (left panels in Fig. 2), the broken disc profile occurs in surface brightness profile only; in the mass density profile the galaxy has a Type I (exponential) disc profile (similar to the galaxy in Fig. 1). Thus for this galaxy, the Type II surface brightness profile is a result of the stellar population gradient, not the mass profile itself. A similar result was found in the simulation by Sánchez-Blázquez et al. (2009).

In the case of Galaxy 971267 (right panels in Fig. 2), it also features a Type II break in its mass density profile as well as its brightness profile. The evolution of the surface density profile for this galaxy is shown in the bottom right panel of Fig. 2. This galaxy has only very minor mergers (stellar mass merger ratios less than 1:10) since $z \sim 2$, and thus the density profile evolution is driven purely by in-situ star formation. The development of a Type II mass profile for this galaxy occurs relatively late in its evolution, from $z \lesssim 0.5$. The density profile for the very outer disc ( $\approx 18 \mathrm{kpc}$ ) has remained nearly constant during this time, with the break radius progressively decreasing as the inner disc $(\sim 5-15 \mathrm{kpc})$ continues to grow. However, Galaxy 971267 appears to be an exception, as most Type II galaxies in the Recal-L025N0752 simulation have near exponential mass profiles. Where a Type II break does occur in the mass profile, it is less pronounced than the break in the light profile (as seen for Galaxy 971267 in Fig. 2).

These results on the origin of Type II disc profiles are in good agreement with that from observed Type II disc galaxies, which also have break radii that coincide with minima in colour, age and $M / L$ (Azzollini et al. 2008a; Bakos et al. 2008; Zheng et al. 2015; RuizLara et al. 2016). Similarly, using $M / L$ profiles to convert surface brightness to mass profiles shows that Type II breaks are far weaker, if present at all, in the mass profiles of observed galaxies (Bakos et al. 2008; Tang et al. 2020). The results from the EAGLE simulations also agree with that of previous simulations, showing that Type II disc truncations result from a drop-off in the cold gas density near the break radius in star-forming galaxies (Roškar et al. 2008; MartínezSerrano et al. 2009; Sánchez-Blázquez et al. 2009).
We note that for barred galaxies the Type II breaks are often coincident with an outer ring structure in the galaxy, likely related to the outer Lindblad resonance of the bars (Pohlen \& Trujillo 2006; Erwin et al. 2008; Muñoz-Mateos et al. 2013). The Type II galaxies from the Recal-L025N0752 volume did not show obvious bars or rings, though the galaxy sample size is also rather small (50 galaxies). Extending this analysis to investigate the connection between bars and Type II breaks in simulated galaxies would be a worthwhile avenue for future work.

\subsection{Type III: anti-truncated disc}

While Type II galaxies in EAGLE largely do not show disc profile breaks in their mass profiles, the case is different for Type III galaxies. We find such anti-truncated discs generally show clear breaks in both their mass and brightness profiles, and thus cannot simply be explained by radial gradients in their stellar populations. Additionally, while Type II galaxies in the EAGLE simulations appear to have a single origin relating to drop-offs in their cold gas/SFR profiles, the origin of Type III profiles is more varied.

For some galaxies, the anti-truncation is a clear result of stars accreted during a galaxy merger (e.g. Bekki 1998; Bournaud et al. 2005), whether a minor or major merger. We show one such example in Fig. 3. This galaxy undergoes a minor merger with a satellite galaxy with $\sim 15$ per cent of its stellar mass at $z \approx 0.1$. Prior to this merger, the galaxy appeared simply to have a Type I (exponential) profile. We do not find any clear indicators in the $M / L$ or age profiles of merger-created Type III galaxies, since it depends upon the relative properties of galaxies involved in the merger and any subsequent star formation. For this example, $M / L$ decreases gradually with increasing radius after peaking at $4 \mathrm{kpc}$. As we will see in Section 4 (Fig. 5), Type III profiles are very common for very massive galaxies with $M_{*}>10^{11} \mathrm{M}_{\odot}$, which build a significant amount of their stellar mass via mergers (Rodriguez-Gomez et al. 2016; Qu et al. 2017; Clauwens et al. 2018; Tacchella et al. 2019; Davison et al. 2020). Anti-truncated profiles formed by mergers may account for the Type III-s profiles (disc embedded within an outer spheroid) observed from some galaxies (Erwin et al. 2005, 2008).

Alternatively, the anti-truncation can be due to an extended starforming disc at late cosmological times. This mechanism is similar to that found by Wang et al. (2018), and related to the up-bending SFR profiles found by Tang et al. (2020). In the left panels of Fig. 4 we show one example, where the anti-truncation is caused by a nearly flat star formation profile at $>10 \mathrm{kpc}$. For this galaxy the inner, steeper profile (radii from 3-10 kpc), is largely in place by $z \sim 0.5$, while the outer, flatter disc profile grows from $z=0.5$ to $z=0$. Interestingly, this outer disc then features a Type II break at $\approx 42 \mathrm{kpc}$, at which point the cold gas surface density begins to drop, but the break is fainter than the adopted surface brightness limit of $27 \mathrm{mag} \mathrm{arcsec}^{-2}$.

In other cases the inverse is true, the inner disc builds up at a later time than the outer disc. We show one case in the right panels of Fig. 4. In this galaxy, the outer disc $(\gtrsim 15 \mathrm{kpc})$ is nearly in place by $z \sim 1$, while the inner disc continues to grow until the present time. This case is reminiscent of Type II galaxies, in that the starforming gas disc drops off significantly beyond $15 \mathrm{kpc}$, but without the corresponding truncation in the brightness profile. Anti-truncated profiles formed by star formation would be expected to correspond to the subset of profiles where the break is a continuation of the disc (Type III-d, Erwin et al. 2005, 2008).

Roediger et al. (2012) and Wang et al. (2018) have also suggested that galaxy harassment in galaxy clusters may play a role in the formation of Type III profiles. However, due to the infrequency of 

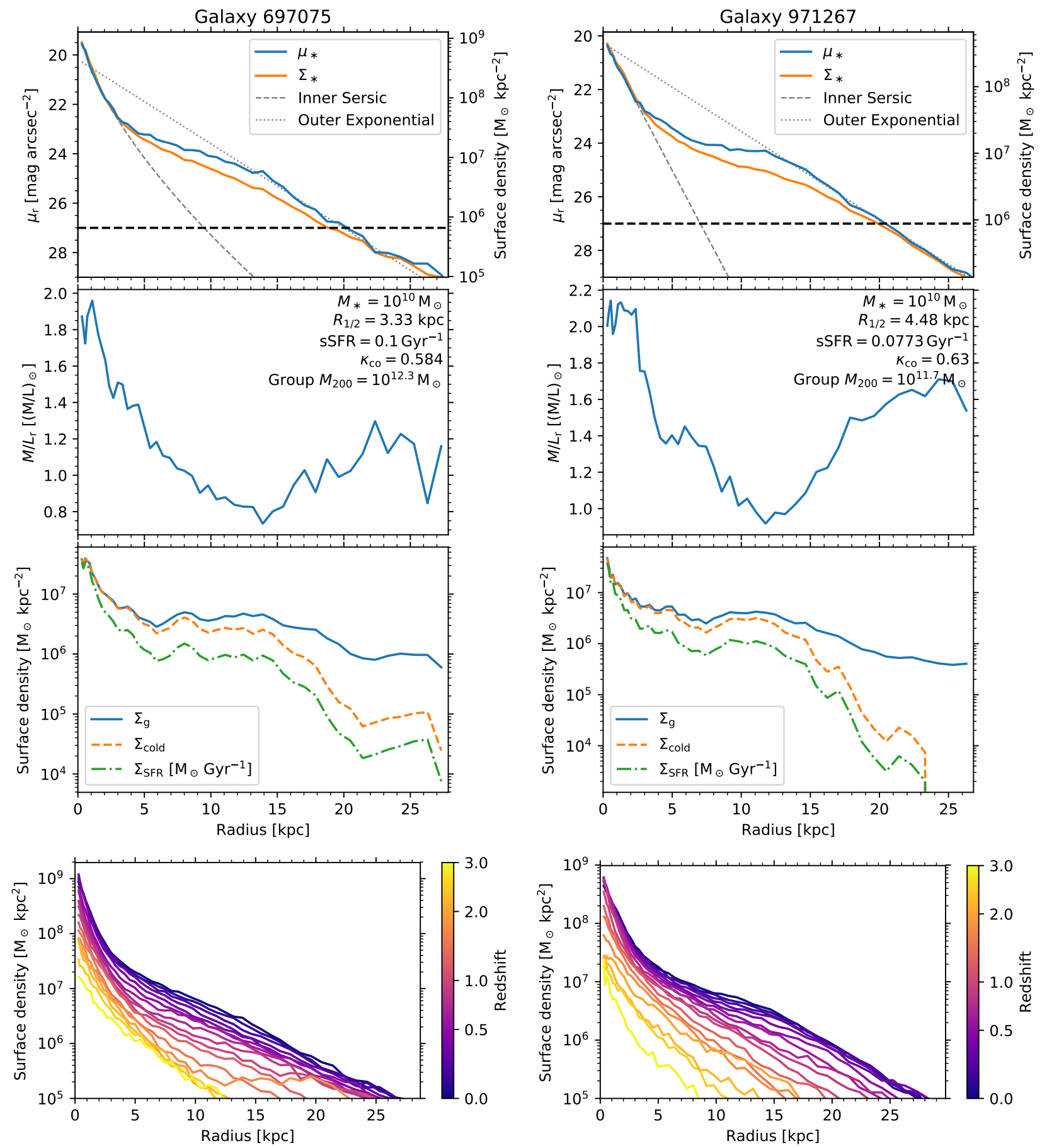

Figure 2. Examples of two Type II galaxies from EAGLE Recal-L025N0752. Panels in the figure are as in Fig. 1. For galaxy 697075 (left panels) the profile truncation mainly exists in the luminosity profile. For galaxy 971267 (right panels) the profile truncation occurs in both the mass and luminosity profiles.

snapshots for the simulations ( 29 between $z=20$ and $z=0$ ), we cannot directly test such an origin. Finally, we note that some processes for Type III profile formation are not exclusive, and thus in some cases more than one effect may be at play within the one galaxy (e.g. accretion of both stars and gas during a merger, where the accreted gas subsequently fuels further star formation in the disc).

\section{EFFECT OF ENVIRONMENT ON DISC PROFILE TYPES}

In this section, we investigate the light profiles of galaxies in the RefL100N1504 simulation, which provides better sampling of galaxies in different environments due to the larger simulation volume (at the expense of poorer sampling of the radial profiles due to the lower resolution). Uncertainties on the frequencies in this section were calculated using binomial statistics and show $\pm 1 \sigma$ uncertainties. 

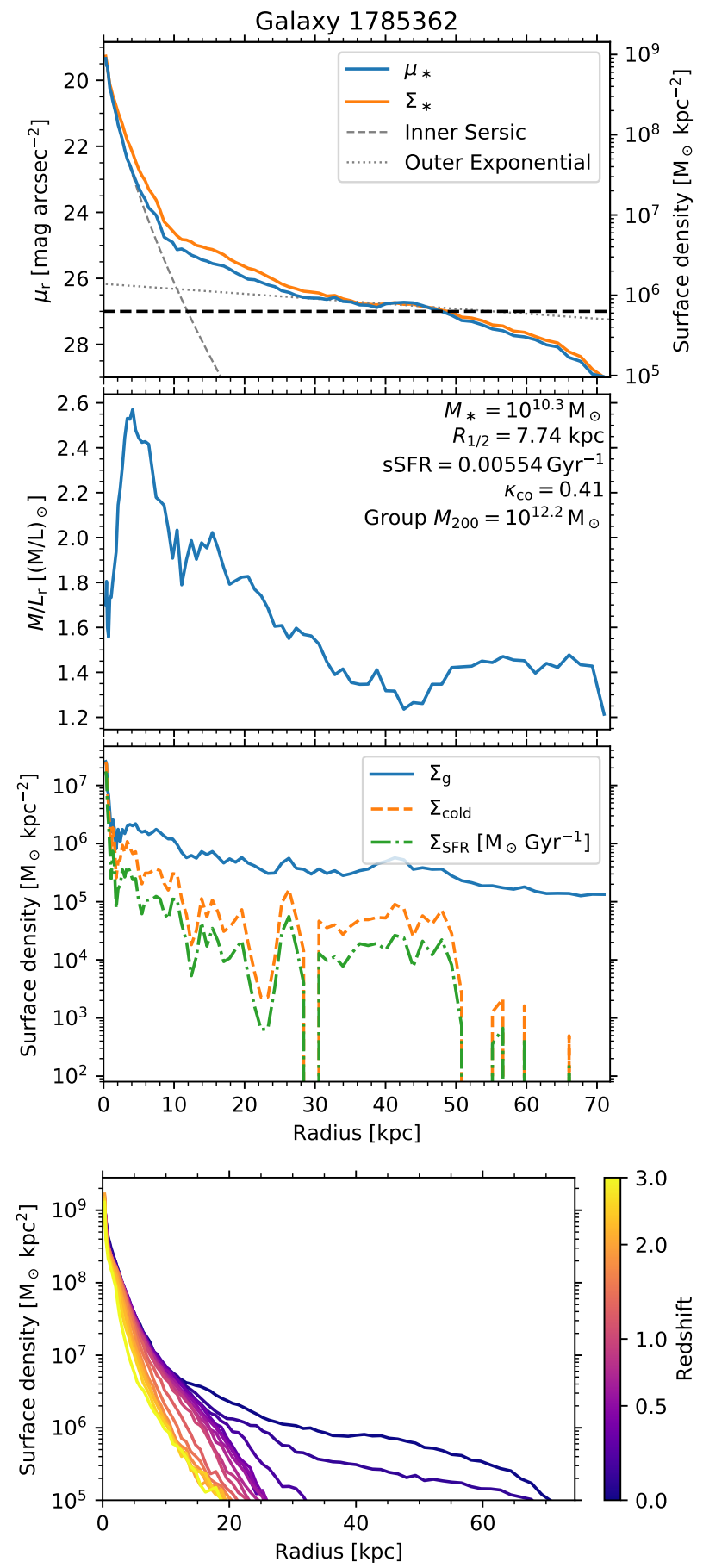

Figure 3. Example of a Type III (anti-truncated) galaxy formed from a late $(z \approx 0.1)$ minor merger (stellar masses $\approx 2 \times 10^{10}$ and $\left.\approx 3 \times 10^{9} \mathrm{M}_{\odot}\right)$. Panels in the figure are as in Fig. 1.

\subsection{Correlations with mass, morphology and environment}

In Fig. 5, we first show the relationship between disc type and galaxy stellar mass for the whole sample of simulated galaxies. Though the exact fractions differ (between both observational studies as well as the simulations), the results are in qualitative agreement with the trends found by Laine et al. (2016) and Tang et al. (2020) showing that the fraction of Type III galaxies increases with galaxy mass, while the fraction of Type I galaxies decreases with increasing galaxy mass. The fraction of Type III galaxies in the simulations shows a steeper trend with mass than the observations. For masses $10^{10.5}$ $10^{11} \mathrm{M}_{\odot}$ the simulations are in good agreement with the Tang et al. (2020) results (Type III fraction $\sim 45$ per cent), but for masses $10^{10}-10^{10.5} \mathrm{M}_{\odot}$ are in better agreement with the Laine et al. (2016) results (Type III fraction $\sim 25$ per cent). As discussed in Section 3.3, the increase of Type III galaxies with galaxy mass can be understood due to the increasing contribution of mergers to galaxy growth, either through the direct accretion of stars creating a profile break, or via the accretion of gas to form extended star-forming discs.

The fraction of Type II galaxies is relatively constant for stellar masses $<10^{11} \mathrm{M}_{\odot}$, at around 30-35 per cent, but drops off significantly at higher masses. This fraction is slightly lower than observed ( $\approx 40$ per cent, Laine et al. 2016; Tang et al. 2020), but is similarly independent of galaxy mass within the mass range $10^{10}-10^{11} \mathrm{M}_{\odot}$. The lower fraction of Type II galaxies in the EAGLE simulations relative to observed galaxies, and thus the slightly higher fraction of Type I galaxies, might potentially be due to the lower than observed star formation rates of EAGLE galaxies (Furlong et al. 2015). However, we note that the fraction of Type II profiles in field galaxies $\left(M_{200} \sim 10^{12} \mathrm{M}_{\odot}\right.$, see Fig. 6) is similar to the observed fractions ( $\sim 0$ per cent).

We find that $4.4_{-0.4}^{+0.5}$ per cent of the simulated galaxies have composite profiles (i.e. Type II+III or III+II), somewhat lower than the $8 \pm 2$ per cent found by Gutiérrez et al. (2011). However the fraction of composite types is dependent on galaxy stellar mass, increasing from $3.1 \pm 0.4$ per cent for $M_{*}<10^{10.75} \mathrm{M}_{\odot}$ to $13 \pm 2$ per cent for $M_{*}>10^{10.75} \mathrm{M}_{\odot}$. Therefore, a galaxy sample weighted to higher masses would obtain a higher fraction of composite profiles. These composite profiles have a number of origins. For Type II+III galaxies, the inner Type II break can be due to a compact, truncated star-forming disc (as in Section 3.2) or a bar (c.f. Erwin et al. 2008). Type III+II galaxies can result from very extended star-forming disc, bearing a relation to Type III galaxies formed via a similar manner (see Section 3.3 and Fig. 4). Of the galaxies with composite types, $73_{-5}^{+4}$ per cent are Type II+III.

In Fig. 6 we compare the relationship between disc profile type and group mass $\left(M_{200}\right.$ of the FOF group in which the galaxy resides at $z=0$ ). The fraction of Type II galaxies shows a clear decrease in denser environments (increasing $M_{200}$ ), consistent with the findings of Erwin et al. (2012) and Roediger et al. (2012). In contrast, the fraction of Type III galaxies increases with group mass (from $10^{12}$ to $10^{13} \mathrm{M}_{\odot}$ ), while the fraction of Type I galaxies is lowest at $M_{200} \sim$ $10^{12.5} \mathrm{M}_{\odot}$ and otherwise remaining at $\approx 40$ per cent at lower and higher group masses. We will further discuss the origin of these trends in Section 4.2.

Next, we investigate the trend of disc profile type with morphology (via the kinematic indicator $\kappa_{\mathrm{co}}$ ) and environment in Fig. 7. Correa et al. (2019) found that $\kappa_{\mathrm{co}}$ correlates reasonably well with visual morphology (see their section 2.3). In this classification, $\mathrm{S} 0$ galaxies have $\kappa_{\mathrm{co}} \sim 0.4$ while Sbc or later corresponds to $\kappa_{\mathrm{co}} \gtrsim 0.6$. We divide the sample by halo mass $\left(M_{200}\right)$ of the FOF group into 'field galaxies' $\left(M_{200}<10^{13} \mathrm{M}_{\odot}\right)$, 'galaxy groups' $\left(10^{13}<M_{200} / \mathrm{M}_{\odot}<10^{14}\right)$ and 'galaxy clusters' $\left(M_{200}>10^{14} \mathrm{M}_{\odot}\right)$. The galaxy cluster sample has similar halo masses to the Virgo cluster $\left(M_{200} \approx 4 \times 10^{14} \mathrm{M}_{\odot}\right.$, McLaughlin 1999).

The trend of disc type with morphology for 'field' galaxies is in good agreement with observations of field galaxies (e.g. Gutiérrez et al. 2011; Tang et al. 2020): the frequency of Type II galaxies increases as galaxies become more disc dominated, while the fraction of Type I and III galaxies decreases with increasing $\kappa_{\mathrm{co}}$.

For S0-like galaxies with $0.35<\kappa_{\mathrm{co}}<0.45$, the fraction of 

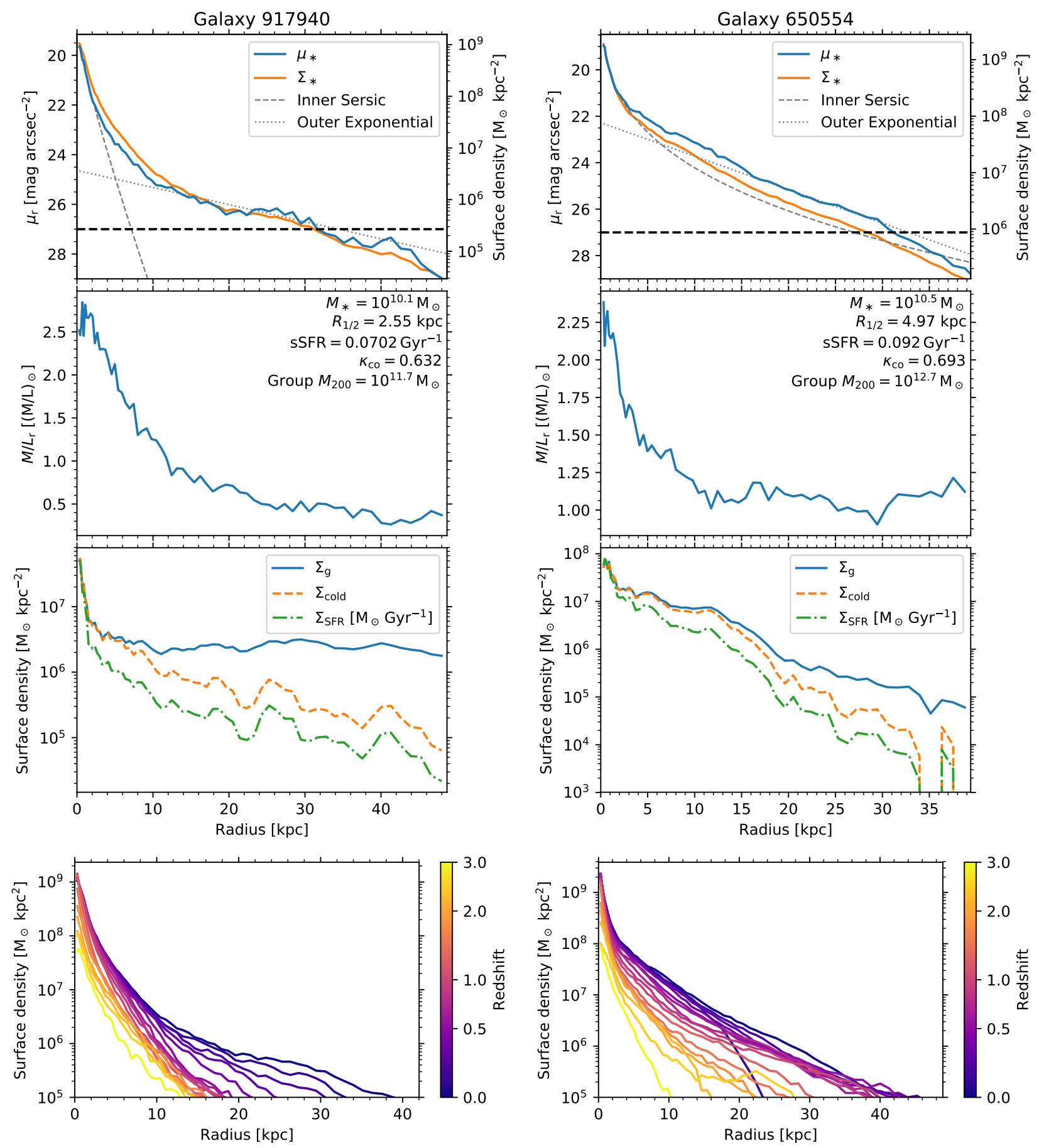

Figure 4. Two examples of Type III profiles from EAGLE Recal-L025N0752 formed via star-forming discs. Panels in the figure are as in Fig. 1. For galaxy 917940 (left panels) the outer disc profile ( $\gtrsim 10 \mathrm{kpc}$ ) builds up late due to an extended star-forming disc. For galaxy 650554 (right panels) the outer disc profile $(15-30 \mathrm{kpc})$ forms prior to the inner disc $(5-15 \mathrm{kpc})$.

Type II galaxies decreases from $22 \pm 2$ per cent in field galaxies, to $6_{-2}^{+3}$ per cent in galaxy clusters. Similarly, for very disc dominated galaxies with $\kappa_{\mathrm{co}}>0.6$ the fraction of Type II galaxies decreases from $61 \pm 2$ per cent in field galaxies, to $21_{-6}^{+7}$ per cent in galaxy clusters. These results are in good agreement with the observations of Erwin et al. (2012) and Roediger et al. (2012), who found a lack of Type II S0 galaxies and a suppressed fraction of Type II disc galaxies in the Virgo cluster, respectively. In the Fornax cluster, a similarly suppressed fraction of Type II disc galaxies (38 per cent) was found by Raj et al. (2019). A number of Fornax cluster S0 galaxies were found to have Type II breaks, largely associated with inner bar structures (Iodice et al. 2019). However, FCC148 hosts a Type II break unassociated with a bar, which would imply a Type II So fraction of $1 / 7 \approx 14_{-9}^{+18}$ per cent (excluding the three edge-on galaxies). Clearly futher work comparing intermediate environments between field galaxies and galaxy clusters is warranted, which would 


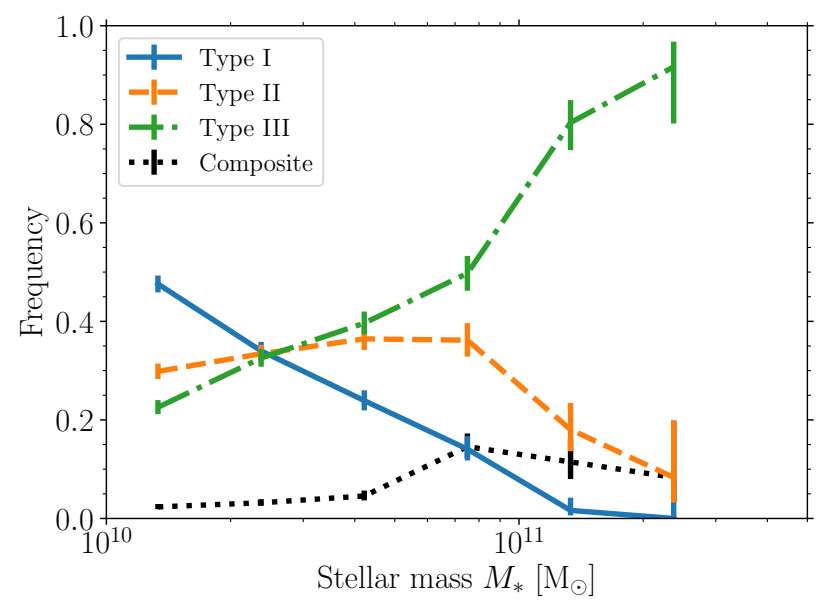

Figure 5. The frequency of galaxy profile types as a function of galaxy stellar mass in the EAGLE Ref-L100N1504 simulation. The fraction of Type I (exponential) profiles decreases with increasing stellar mass, while the fraction of and Type III (anti-truncated) profiles increases with stellar mass, in agreement with observed galaxies (Laine et al. 2016; Tang et al. 2020). The black dotted line shows the fraction of composite type profiles (Type II+III or III+II). Errorbars show the $1 \sigma$ uncertainties from binomial statistics.

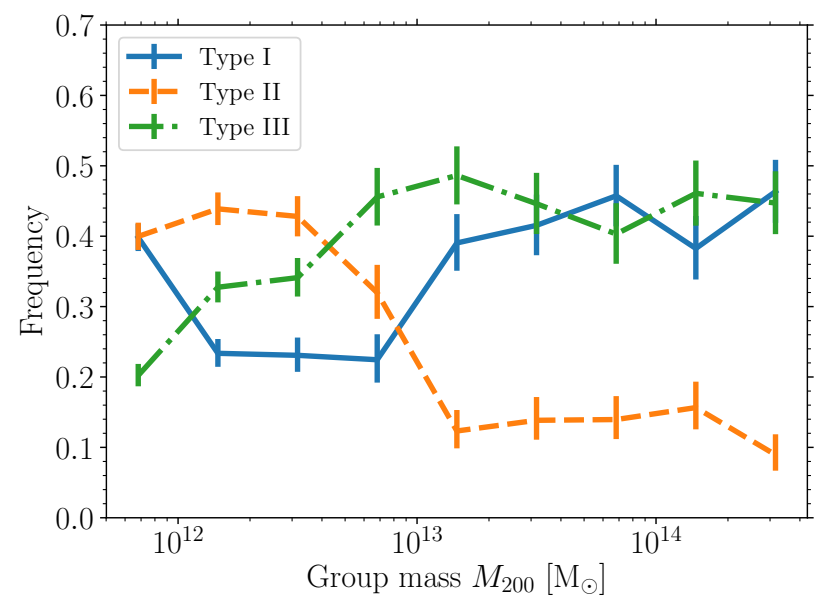

Figure 6. The frequency of galaxy disc types as a function of group mass $\left(M_{200}\right)$ in the EAGLE Ref-L100N1504 simulation. The fraction of Type II galaxies is highest in 'field' galaxies $\left(M_{200}<10^{13} \mathrm{M}_{\odot}\right)$, then drops of significantly in groups with $M_{200}>10^{13} \mathrm{M}_{\odot}$. Errorbars show the $1 \sigma$ uncertainties from binomial statistics.

enable the predictions from the simulations to be tested in more detail.

The EAGLE simulations qualitatively reproduce the observed trends found between disc profile types and galaxy mass, morphology and environment, meaning we can now use them investigate the origin of the lower Type II frequencies in galaxy clusters.

\subsection{The lower incidence of Type II profiles in galaxy clusters}

The results of Section 3.2 show that Type II profile depend strongly on active/recent star formation in the galaxy. Therefore in this section we investigate the dependence of disc profiles on the SFRs of the galaxies. When determining the SFRs of the simulated galaxies, we use the instantaneous SFR derived from the gas particle (i.e. the sum of all instantaneous SFRs for each star formation-eligible gas particle in the galaxy).

In Fig. 8 we show the frequency of disc profile types as a function of the specific star formation rate $\left(\mathrm{SSFR}=\mathrm{SFR} / M_{*}\right.$ ) of the galaxies. There is a clear relationship with sSFR for Type II profile galaxies: $48 \pm 1$ per cent of galaxies on the main star-forming sequence (sSFR > $10^{-1.5} \mathrm{Gyr}^{-1}$ ) have Type II profiles, while only $5 \pm 1$ per cent of quiescent galaxies (sSFR $\lesssim 10^{-2.5} \mathrm{Gyr}^{-1}$ ) have Type II profiles. Instead, non-star forming galaxies are divided into Type I and III profiles in roughly equal manner ( $47 \pm 3$ per cent).

The relationship between Type II profiles and sSFR is easily understood from the findings in Section 3.2. The majority of Type II disc profiles in the EAGLE simulations are a result of truncated star-forming discs, which drive radial changes in the stellar mass-tolight ratios that cause breaks in the surface brightness profiles. Thus, galaxies without on-going or recent star formation generally cannot have Type II profiles. The relationship between Type II profiles and sSFR also explains the increasing fraction of Type II galaxies with Hubble type (Fig. 7; Pohlen \& Trujillo 2006; Gutiérrez et al. 2011; Laine et al. 2016; Tang et al. 2020), given the relationship between Hubble type and SFR (e.g. Kennicutt 1998).

This finding thus provides a natural explanation for the lack of Type II S0 galaxies (Erwin et al. 2012) and the suppressed fraction of Type II disc galaxies (which decrease from a fraction of 50-60 percent to 34 percent, Roediger et al. 2012) in the Virgo cluster, given that galaxies in denser environments show reduced sSFRs (e.g. Lewis et al. 2002; Kauffmann et al. 2004; Darvish et al. 2016). In Fig. 9 we show the fraction of star-forming galaxies (with sSFR > $\left.10^{-1.5} \mathrm{Gyr}^{-1}\right)^{1}$ as a function of the group mass $\left(M_{200}\right)$ and separated by the morphology $\left(\kappa_{\mathrm{co}}\right)$ of the galaxies. The kinematic morphology $\kappa_{\mathrm{co}}$ and SFR are roughly correlated, such that high- $\kappa_{\mathrm{co}}$ galaxies tend to be blue and star forming, while low- $\kappa_{\text {co }}$ galaxies tend to be red and quenched (Correa et al. 2017, 2019). In 'field' galaxies $\left(M_{200} \sim 10^{12} \mathrm{M}_{\odot}\right)$ the majority of galaxies are star forming. This fraction decreases with increasing $M_{200}$, such that nearly all S0-like galaxies $\left(0.35<\kappa_{\mathrm{co}}<0.45\right)$ and over half of very disc dominated galaxies $\left(\kappa_{\mathrm{co}}>0.6\right)$ in galaxy clusters $\left(M_{200}>10^{14} \mathrm{M}_{\odot}\right)$ are nonstar forming. Combined with the very low fraction of Type II galaxies with sSFR $<10^{-1.5} \mathrm{Gyr}^{-1}$ (Fig. 8), this result explains the absence of Type II S0 galaxies (Erwin et al. 2012) and the lower fraction of Type II disc galaxies (Roediger et al. 2012) in the Virgo cluster.

Conversely, our results suggest a connection to recent star formation for the $28_{-6}^{+7}$ per cent of field S0 galaxies with Type II profiles (Erwin et al. 2012). This may be problematic, given S0 galaxies are typically not found on the star-forming main sequence (e.g. Bait et al. 2017; Mishra et al. 2019). Comparing the galaxy catalogues of Bait et al. (2017) and Mishra et al. (2019), and limiting to galaxies with stellar masses $10^{10}<M_{*} / \mathrm{M}_{\odot}<10^{10.5}$ (the most relevant comparison for the volume-limit EAGLE galaxy sample), shows that in field environments (galaxy number density $\Sigma<10^{-0.5} \mathrm{Mpc}^{-2}$ ) only 25 35 per cent of S0 galaxies are star forming ${ }^{2}$, which would imply that essentially all star-forming S0 galaxies must be Type II. Fig 8 suggests that, at maximum, around 50 per cent of star-forming galaxies would be expected to have Type II profiles, indicating some tension

${ }^{1}$ At stellar masses $M_{*} \sim 10^{10} \mathrm{M}_{\odot}$, the limit of sSFR $>10^{-1.5} \mathrm{Gyr}^{-1}$ encompasses approximately 90 per cent of 'star-forming' EAGLE galaxies (Furlong et al. 2015).

2 The Bait et al. (2017) catalogue, which derives SFRs by modelling the spectral energy distribution, shows a higher fraction of star-forming galaxies than the catalogue from Mishra et al. (2019), which use NUV-r colours to categorise the galaxies. 

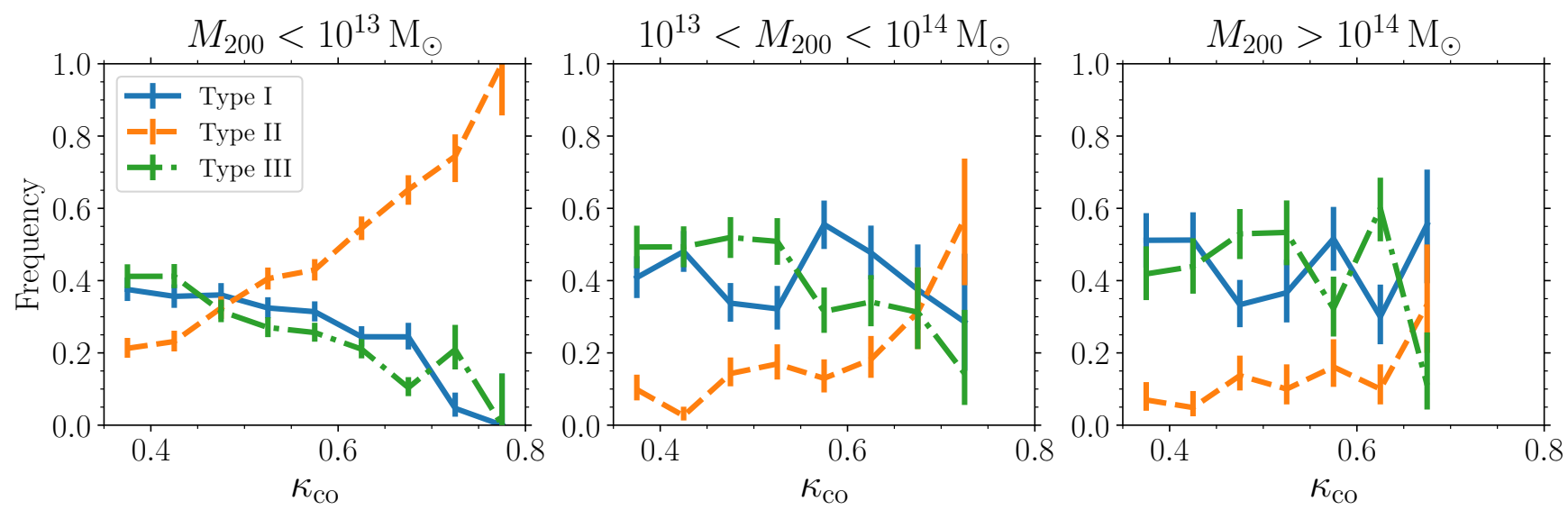

Figure 7. Frequency of galaxy disc types as a function of kinematic morphology, $\kappa_{\mathrm{co}}$ (fraction of stellar kinetic energy invested in ordered co-rotation). In this classification, S0 galaxies have $\kappa_{\mathrm{co}} \sim 0.4$ and Sbc or later corresponds to $\kappa_{\mathrm{co}} \gtrsim 0.6$. The galaxy sample is split into three different environments: 'field galaxies' $\left(M_{200}<10^{13} \mathrm{M}_{\odot}\right)$, 'galaxy groups' $\left(10^{13}<M_{200} / \mathrm{M}_{\odot}<10^{14}\right)$ and 'galaxy clusters' $\left(M_{200}>10^{14} \mathrm{M}_{\odot}\right)$. Errorbars show the $1 \sigma$ uncertainties from binomial statistics.

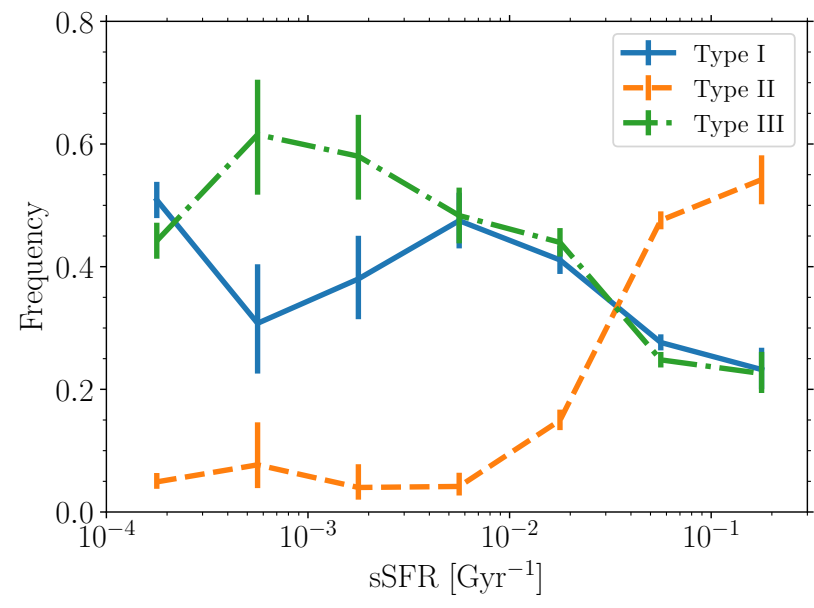

Figure 8. Frequency of disc profiles types relative to the specific star formation rates $\left(\mathrm{SFR} / M_{*}\right)$ of galaxies in the EAGLE Ref-L100N1504 simulation. Galaxies with sSFR $<10^{-4} \mathrm{Gyr}$ are included in the lowest sSFR bin. Almost no Type II profiles are found for galaxies with low sSFRs $\left(<10^{-2} \mathrm{Gyr}^{-1}\right)$. Errorbars show the $1 \sigma$ uncertainties from binomial statistics.

between the simulation and observation results unless the fraction of star-forming S0 galaxies with Type II profiles is far higher, or 'green valley' galaxies also contribute to the Type II S0 population (i.e. galaxies with SFRs below the main star-forming sequence but which are not completely quenched of star formation; 20-30 per cent of the S0 population are green valley galaxies, Bait et al. 2017).

Erwin et al. (2008) found that many of the Type II S0 galaxies have a break that coincides with an outer ring. Such structures are common in lenticular galaxies, where around 40 per cent have outer rings (Comerón et al. 2014). Some outer rings, and the corresponding Type II breaks, may be related to the dynamics of bars (in particular the outer Lindblad resonance, Pohlen \& Trujillo 2006; Erwin et al. 2008; Muñoz-Mateos et al. 2013), though rings also occur in nonbarred galaxies which might be caused by tidal interactions (for a review on the formation of rings in galaxies, see Buta \& Combes 1996). In many cases the outer rings of galaxies are UV-bright (over 50 per

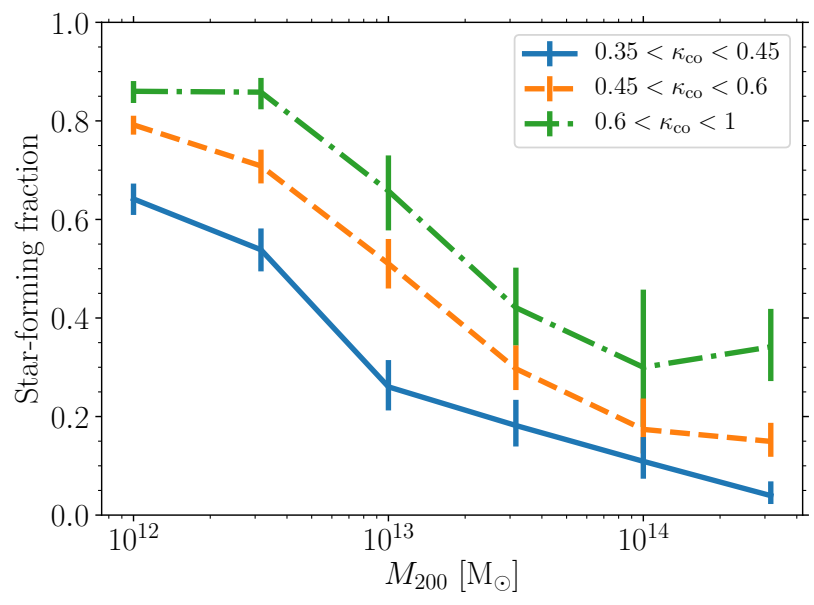

Figure 9. Fraction of EAGLE galaxies with sSFR $>0.03 \mathrm{Gyr}^{-1}$ (i.e. starforming galaxies) compared with the galactic environment $\left(M_{200}\right)$. The galaxy sample is divided based on the kinematic morphology $\kappa_{\mathrm{co}}$. The fraction of star-forming galaxies decreases consistently with increasing $\boldsymbol{M}_{200}$ across all galaxy morphologies. Errorbars show the $1 \sigma$ uncertainties from binomial statistics.

cent in S0 galaxies), indicating ongoing star formation (Kostiuk \& Sil'chenko 2015; Proshina et al. 2019). Whether bars and/or rings in S0 galaxies result in a higher proportion of star-forming galaxies with Type II profiles, or if some other process is at work, is unclear, and we intend to follow up this topic in future work.

\subsection{What were the disc types of $\mathrm{S} 0$ galaxies prior to cluster infall?}

The absence of Type II S0 galaxies in galaxy clusters leads to the question of whether the profiles were transformed within the galaxy clusters, or if Type II profiles were already absent prior to the galaxies entering clusters. For galaxies with $0.35<\kappa_{\text {co }}<0.45$ (S0-like morphologies) and in galaxy clusters with $M_{200}>10^{14} \mathrm{M}_{\odot}$ at $z=0$, we classified their $r$-band surface brightness profiles at the snapshot 


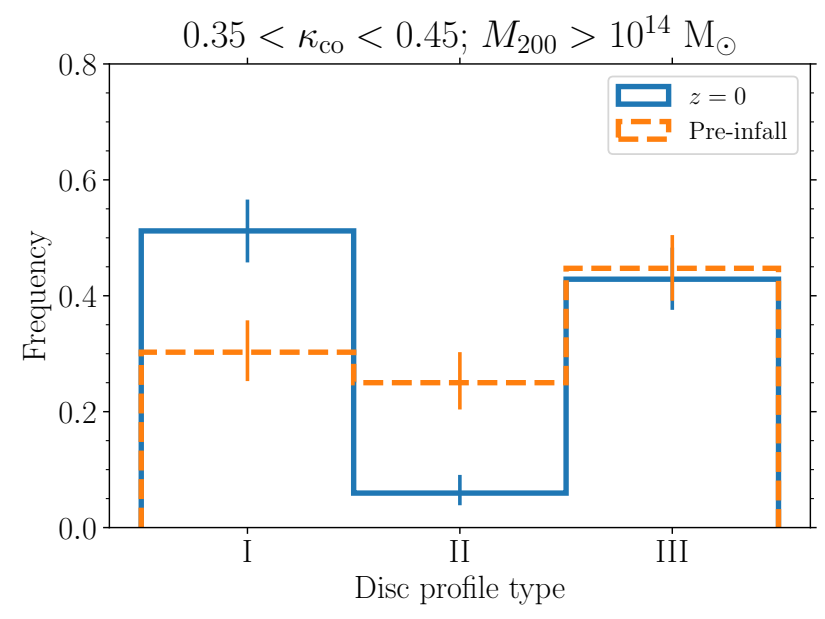

Figure 10. The frequency of disc profile types for S0-like galaxies $(0.35<$ $\left.\kappa_{\mathrm{co}}<0.45\right)$ in galaxy clusters $\left(M_{200}>10^{14} \mathrm{M}_{\odot}\right)$ at the present day $(z=0)$ and prior to becoming satellite galaxies (pre-infall). Errorbars show the $1 \sigma$ uncertainties from binomial statistics.

prior to them becoming satellite galaxies (i.e. the last snapshot at which the galaxies were considered central subhaloes in their FOF groups).

We compare the frequencies of the pre-infall disc types with the $z=0$ disc types for these galaxies in Fig.10. Overall, the frequency of pre-infall disc types $(30 \pm 5,25 \pm 5$ and $45 \pm 6$ per cent for Types I, II and III, respectively) is relatively similar to the frequency of present day S0-like disc types for field galaxies $(37 \pm 2,22 \pm 2$ and $41 \pm 2$ per cent; Fig. 7). The fraction of Type III galaxies is similar both before infall ( $45 \pm 6$ per cent $)$ and at $z=0(43 \pm 5$ per cent $)$, indicating antitruncated profiles are largely unaffected by the cluster environment.

However, the fraction of Type II galaxies decreases from $25 \pm 5$ per cent pre-infall to $6_{-2}^{+3}$ per cent at $z=0$. The difference is made up by an increase in the Type I fraction at $z=0$, implying that the young stellar populations of Type II galaxies simply fade to become Type I galaxies upon cessation of star formation within the cluster environment (e.g. through ram pressure stripping, Gunn \& Gott 1972, and/or starvation, Larson et al. 1980). This finding is in good agreement with observations of Virgo cluster S0 galaxies, where the lack of Type II profiles is similarly made up by an increased fraction of Type I profiles relative to field galaxies (Erwin et al. 2012).

\section{SUMMARY}

In this work, we investigated the origin of disc profile breaks and their dependence on galaxy properties and galactic environment in the EAGLE simulations. Following observational works, we classified $r$-band surface brightness profiles of the simulated galaxies into exponential (Type I), truncated (Type II) and anti-truncated (Type III) discs.

In Section 3 we investigated the origins of the disc profile breaks. We found, in agreement with previous observational and theoretical work (Sánchez-Blázquez et al. 2009), that Type II (truncated) discs predominantly result from truncated star-forming discs (Section 3.2). The truncated star-forming discs cause radial gradients in the stellar populations, which in turn (through $M / L$ variations), drive breaks in the surface brightness profiles that are not necessarily found in the underlying mass profiles (see also Bakos et al. 2008; Sánchez-Blázquez et al. 2009; Tang et al. 2020). In the EAGLE model, the truncated star- forming discs result from density thresholds for star formation and occur when the total gas density falls below $\sim 10^{6.5} \mathrm{M}_{\odot} \mathrm{kpc}^{-2}$ (see Schaye 2004; Schaye \& Dalla Vecchia 2008). Upon entering galaxy groups and clusters, and following a cessation in star formation, in most cases the young stellar populations of Type II galaxies simply fade, leaving behind Type I galaxies (Section 4.3). This agrees well with observational evidence suggesting S0 galaxies in large groups are predominantly faded spiral galaxies (Deeley et al. 2020) and simulations showing S0-progenitor galaxies lose their gas soon after group/cluster infall (Deeley et al., MNRAS, submitted).

In contrast, we found that Type III (anti-truncated) discs have a more varied origin (Section 3.3). The profile breaks are also present in the underlying mass profiles, thus are not driven by stellar population gradients like for Type II discs. In the simulations we found examples of anti-truncations created through galaxy mergers (both major and minor mergers, extended star-forming discs and the late build-up of a steeper inner disc. The increasing importance of mergers for stellar mass growth in massive galaxies (e.g. Rodriguez-Gomez et al. 2016; Qu et al. 2017) suggests that the increasing fraction of Type III galaxies with galaxy mass (Fig. 5) is driven by galaxy mergers.

In Section 4 we investigated the origin of the lower incidence (relative to field galaxies) of Type II galaxies in galaxy clusters (Erwin et al. 2012; Roediger et al. 2012; Raj et al. 2019). In agreement with observational studies, we found in the simulations that almost no S0like galaxies in galaxy clusters have Type II profiles, while the fraction of Type II profiles for disc-dominated galaxies also significantly decreases (Fig. 7). We found that this decrease in Type II fraction can be directly attributed to the lower SFRs of galaxies residing in clusters: the frequency of Type II profiles is a strong function of the galaxies' specific SFR (Fig. 8) and the fraction of star-forming galaxies is a strong function of group/cluster mass (Fig. 9). Therefore, low SFR galaxies in galaxy clusters generally do not host Type II profiles, unlike their star-forming morphological analogues in the field environment.

\section{ACKNOWLEDGEMENTS}

We thank Michael Drinkwater and Camila Correa for helpful comments and discussion, and the referee for a very constructive report which improved the paper. This research was supported by the Australian government through the Australian Research Council's Discovery Projects funding scheme (DP200102574). This work used the DiRAC Data Centric system at Durham University, operated by the Institute for Computational Cosmology on behalf of the STFC DiRAC HPC Facility (www.dirac.ac.uk). This equipment was funded by BIS National E-infrastructure capital grant ST/K00042X/1, STFC capital grants ST/H008519/1 and ST/K00087X/1, STFC DiRAC Operations grant ST/K003267/1 and Durham University. DiRAC is part of the National E-Infrastructure.

\section{DATA AVAILABILITY}

All data (including galaxy catalogues, merger trees and particle data) from the EAGLE simulations is publicly available (McAlpine et al. 2016) at http://www.eaglesim.org/database.php.

\section{REFERENCES}

Azzollini R., Trujillo I., Beckman J. E., 2008a, ApJ, 679, L69

Azzollini R., Trujillo I., Beckman J. E., 2008b, ApJ, 684, 1026 
Bahé Y. M., et al., 2016, MNRAS, 456, 1115

Bait O., Barway S., Wadadekar Y., 2017, MNRAS, 471, 2687

Bakos J., Trujillo I., Pohlen M., 2008, ApJ, 683, L103

Bekki K., 1998, ApJ, 502, L133

Bignone L. A., Pedrosa S. E., Trayford J. W., Tissera P. B., Pellizza L. J., 2020, MNRAS, 491, 3624

Booth C. M., Schaye J., 2009, MNRAS, 398, 53

Borlaff A., et al., 2018, A\&A, 615, A26

Bournaud F., Jog C. J., Combes F., 2005, A\&A, 437, 69

Buta R., Combes F., 1996, Fundamentals Cosmic Phys., 17, 95

Chabrier G., 2003, PASP, 115, 763

Charlot S., Fall S. M., 2000, ApJ, 539, 718

Clauwens B., Schaye J., Franx M., Bower R. G., 2018, MNRAS, 478, 3994

Comerón S., et al., 2014, A\&A, 562, A121

Conroy C., Gunn J. E., 2010, ApJ, 712, 833

Conroy C., Gunn J. E., White M., 2009, ApJ, 699, 486

Correa C. A., Schaye J., 2020, MNRAS, 499, 3578

Correa C. A., Schaye J., Clauwens B., Bower R. G., Crain R. A., Schaller M., Theuns T., Thob A. C. R., 2017, MNRAS, 472, L45

Correa C. A., Schaye J., Trayford J. W., 2019, MNRAS, 484, 4401

Crain R. A., et al., 2015, MNRAS, 450, 1937

Crain R. A., et al., 2017, MNRAS, 464, 4204

Dalla Vecchia C., Schaye J., 2012, MNRAS, 426, 140

Darvish B., Mobasher B., Sobral D., Rettura A., Scoville N., Faisst A., Capak P., 2016, ApJ, 825, 113

Davis M., Efstathiou G., Frenk C. S., White S. D. M., 1985, ApJ, 292, 371

Davison T. A., Norris M. A., Pfeffer J. L., Davies J. J., Crain R. A., 2020, MNRAS, 497, 81

Deeley S., et al., 2020, MNRAS, 498, 2372

Dolag K., Borgani S., Murante G., Springel V., 2009, MNRAS, 399, 497

Erwin P., Beckman J. E., Pohlen M., 2005, ApJ, 626, L81

Erwin P., Pohlen M., Beckman J. E., 2008, AJ, 135, 20

Erwin P., Gutiérrez L., Beckman J. E., 2012, ApJ, 744, L11

Freeman K. C., 1970, ApJ, 160, 811

Furlong M., et al., 2015, MNRAS, 450, 4486

Furlong M., et al., 2017, MNRAS, 465, 722

Girardi L., Bressan A., Bertelli G., Chiosi C., 2000, A\&AS, 141, 371

Gunn J. E., Gott J. Richard I., 1972, ApJ, 176, 1

Gutiérrez L., Erwin P., Aladro R., Beckman J. E., 2011, AJ, 142, 145

Herpich J., Stinson G. S., Rix H. W., Martig M., Dutton A. A., 2017, MNRAS, 470, 4941

Iodice E., et al., 2019, A\&A, 623, A1

Kauffmann G., White S. D. M., Heckman T. M., Ménard B., Brinchmann J., Charlot S., Tremonti C., Brinkmann J., 2004, MNRAS, 353, 713

Kennicutt Robert C. J., 1989, ApJ, 344, 685

Kennicutt Robert C. J., 1998, ARA\&A, 36, 189

Knebe A., et al., 2011, MNRAS, 415, 2293

Kostiuk I. P., Sil'chenko O. K., 2015, Astrophysical Bulletin, 70, 280

Lagos C. d. P., et al., 2015, MNRAS, 452, 3815

Lagos C. d. P., et al., 2016, MNRAS, 459, 2632

Laine J., et al., 2014, MNRAS, 441, 1992

Laine J., Laurikainen E., Salo H., 2016, A\&A, 596, A25

Larson R. B., Tinsley B. M., Caldwell C. N., 1980, ApJ, 237, 692

Lee Y., Park H. S., Kim S. C., Moon D.-S., Lee J.-J., Kim D.-J., Cha S.-M., 2018, ApJ, 859, 5

Lewis I., et al., 2002, MNRAS, 334, 673

Marasco A., Crain R. A., Schaye J., Bahé Y. M., van der Hulst T., Theuns T., Bower R. G., 2016, MNRAS, 461, 2630

Marigo P., Girardi L., 2007, A\&A, 469, 239

Marigo P., Girardi L., Bressan A., Groenewegen M. A. T., Silva L., Granato G. L., 2008, A\&A, 482, 883

Martínez-Serrano F. J., Serna A., Doménech-Moral M., Domínguez-Tenreiro R., 2009, ApJ, 705, L133

McAlpine S., et al., 2016, Astronomy and Computing, 15, 72

McLaughlin D. E., 1999, ApJ, 512, L9

Méndez-Abreu J., et al., 2017, A\&A, 598, A32

Mishra P. K., Wadadekar Y., Barway S., 2019, MNRAS, 487, 5572

Muñoz-Mateos J. C., et al., 2013, ApJ, 771, 59
Muldrew S. I., Pearce F. R., Power C., 2011, MNRAS, 410, 2617

Oppenheimer B. D., et al., 2016, MNRAS, 460, 2157

Patterson F. S., 1940, Harvard College Observatory Bulletin, 914, 9

Planck Collaboration 2014, A\&A, 571, A16

Pohlen M., Trujillo I., 2006, A\&A, 454, 759

Proshina I. S., Kniazev A. Y., Sil'chenko O. K., 2019, AJ, 158, 5

Qu Y., et al., 2017, MNRAS, 464, 1659

Rahmati A., Schaye J., Bower R. G., Crain R. A., Furlong M., Schaller M., Theuns T., 2015, MNRAS, 452, 2034

Rahmati A., Schaye J., Crain R. A., Oppenheimer B. D., Schaller M., Theuns T., 2016, MNRAS, 459, 310

Raj M. A., et al., 2019, A\&A, 628, A4

Rodriguez-Gomez V., et al., 2016, MNRAS, 458, 2371

Roediger J. C., Courteau S., Sánchez-Blázquez P., McDonald M., 2012, ApJ, 758,41

Rosas-Guevara Y. M., et al., 2015, MNRAS, 454, 1038

Roškar R., Debattista V. P., Stinson G. S., Quinn T. R., Kaufmann T., Wadsley J., 2008, ApJ, 675, L65

Ruiz-Lara T., et al., 2016, MNRAS, 456, L35

Sánchez-Blázquez P., et al., 2006, MNRAS, 371, 703

Sánchez-Blázquez P., Courty S., Gibson B. K., Brook C. B., 2009, MNRAS, 398,591

Schaye J., 2004, ApJ, 609, 667

Schaye J., Dalla Vecchia C., 2008, MNRAS, 383, 1210

Schaye J., et al., 2015, MNRAS, 446, 521

Sérsic J. L., 1963, Boletin de la Asociacion Argentina de Astronomia La Plata Argentina, 6, 41

Sil'chenko O. K., Kniazev A. Y., Chudakova E. M., 2018, AJ, 156, 118

Springel V., 2005, MNRAS, 364, 1105

Springel V., White S. D. M., Tormen G., Kauffmann G., 2001, MNRAS, 328, 726

Tacchella S., et al., 2019, MNRAS, 487, 5416

Tang Y., et al., 2020, ApJ, 897, 79

Thob A. C. R., et al., 2019, MNRAS, 485, 972

Trayford J. W., et al., 2015, MNRAS, 452, 2879

Trayford J. W., et al., 2017, MNRAS, 470, 771

Trujillo I., Pohlen M., 2005, ApJ, 630, L17

Turner M. L., Schaye J., Crain R. A., Theuns T., Wendt M., 2016, MNRAS, 462,2440

Turner M. L., Schaye J., Crain R. A., Rudie G., Steidel C. C., Strom A., Theuns T., 2017, MNRAS, 471, 690

Wang J., et al., 2018, MNRAS, 479, 4292

Watkins A. E., Laine J., Comerón S., Janz J., Salo H., 2019, A\&A, 625, A36

Wiersma R. P. C., Schaye J., Smith B. D., 2009a, MNRAS, 393, 99

Wiersma R. P. C., Schaye J., Theuns T., Dalla Vecchia C., Tornatore L., 2009b, MNRAS, 399, 574

Zheng Z., et al., 2015, ApJ, 800, 120

de Vaucouleurs G., 1959, ApJ, 130, 728

van der Kruit P. C., 1979, A\&AS, 38, 15

This paper has been typeset from a $\mathrm{TE}_{\mathrm{E}} / \mathrm{LAT} \mathrm{E}$ file prepared by the author. 